\title{
The use of CPC theory for energy description of two nonlinear receivers
}

\author{
Konrad Zajkowski ${ }^{1,}{ }^{*}$, Ivan Rusica ${ }^{2}$ and Zuzana Palkova ${ }^{3}$ \\ ${ }^{1}$ Koszalin University of Technology, Department of Mechanical Engineering, 15-17 Raclawicka St., \\ 75-620 Koszalin, Poland \\ ${ }^{2}$ Technical University of Moldova, Technology of Machine Building, Republic of Moldova \\ ${ }^{3}$ Slovak University of Agriculture in Nitra, Trieda A. Hlinku 2, 94976 - Nitra, Slovakia
}

\begin{abstract}
The article concerns a mathematical description of electric power in the case of two nonlinear receivers connected to a common supply network. This description was made on the basis of the fundamental CPC (Currents' Physical Components) power theory. This theory was developed for one nonlinear receiver. In the case of two nonlinear receivers of the same type, i.e. when the harmonics generated by them are created equal, the CPC theory requires some modification. This issue is important when it is necessary to determine the components of currents in an energy system supplying two nonlinear receivers.
\end{abstract}

\section{Introduction}

The power theory is a science that is in the area of interest of many researchers. Until today, there has been no universal power theory, one that would be correct for all the types of systems. A theory based on a description of components, dependent on physical quantities, which can be explained by appropriate phenomena, was presented by Czarnecki [1]. In [2], the author presented a CPC theory for the case of a nonlinear receiver supplied with AC from a real source. This circuit is characterized by the transmission of harmonics that are generated by a nonlinear receiver. Then, taking into account the internal impedance of the source, these harmonics are transferred to other receivers.

\section{Source with one harmonic}

According to [2], the energy phenomena accompanying the generation of harmonics by the receiver will further be marked with the " $C$ " index (Customer load), and from the energy system, with the " $D$ " index (Distribution system).

The considerations presented at this point will refer to the case when the nonlinear receiver is forced by a sinusoidal supply voltage with the fundamental frequency $(n=1)$, and a non-sinusoidal current waveform is a consequence of the phenomena occurring in the receiver [3-11]. The harmonics that belong to the $N_{c}$ group come from the nonlinear receiver.

\footnotetext{
*Corresponding author: 1. konrad.zajkowski@tu.koszalin.pl
} 


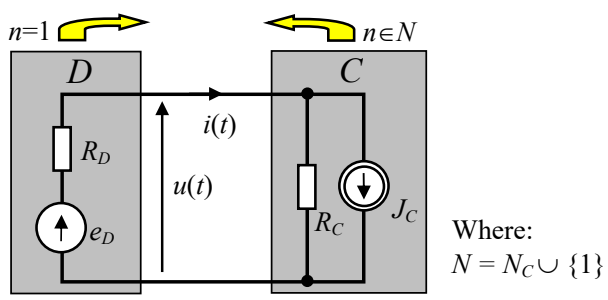

Fig. 1. Cooperation of a nonlinear receiver $(C)$ with a real sinusoidal source $(D)$.

The nonlinear receiver is a higher harmonic generator $\omega_{(n)}$ in the current $i(t)[8,9]$. In addition, when the internal resistance of the source is different from zero $\left(R_{D} \neq 0\right)$, these harmonics are also transferred to the $u(t)$ voltage waveform. The set of harmonics which was created in the receiver in Figure 1 is denoted by $N_{C}$ and is a subset of all $N$ harmonics.

For a resistive-type receiver, the $i_{\mathrm{r}}$ reactive current component is equal to zero. Also, the scattering current component $i_{\mathrm{s}}=0$, because the conductance of the receiver does not change with the frequency.

The active current component $i_{\mathrm{a}}$ for the pulsation $\omega_{1}$ depends from an effective value of the voltage source $E_{D(1)}$, for the remaining pulsations $\omega_{(n)}\left(n \in N_{C}\right)$ depends from the current source $J_{C(n)}$.

Therefore, the powers in this system, in accordance with the CPC theory, are equal: reactive power: $Q=\|u\| \cdot\left\|i_{\mathrm{r}}\right\|=0$, scattering power: $D_{\mathrm{s}}=\|u\| \cdot\left\|i_{\mathrm{s}}\right\|=0$, and active power:

$$
P=\underbrace{U_{(1)} I_{(1)} \cos \varphi_{(1)}}_{P_{D}}+\underbrace{\sum_{n \in N_{C}} U_{(n)} I_{(n)} \cos \varphi_{(n)}}_{P_{C}} .
$$

The active power are consists of two components:

- $\quad P_{D}-$ a component produced by the $E_{D(1)}$ voltage source and lost on the $R_{C}$ resistance of the receiver (the first component of Formula (1)), and

- $\quad P_{C}$ - a component produced by the $J_{C(n)}$ current source and lost on the $R_{D}$ resistance (the second component).

The $P_{D}$ component has a positive value due to the energy flow from the source to the receiver, while the $P_{C}$ takes a negative value due to the change of the voltage sign $U_{(n)}$ caused by the change of the direction of the current in the $R_{C}$ resistance.

The presence of energy flowing in the opposite direction (from the receiver to the source, $n \in N_{C}$ ) was proposed by Czarnecki to be considered as a separate physical phenomenon, one that requires a separate description [12].

The energy returned to the source is useless for the power system, because this energy has pulsation other than $\omega_{1}$. This system is additionally occupied by a current with harmonics that are not used by the system. The transmission capacity of the network is smaller for the current with $\omega_{1}$ pulsation.

\section{A source with many harmonics}

A case where a nonlinear receiver is powered from a real source with many harmonics is considered here.

The set of harmonics marked with $N_{D}$ corresponds to the pulsations of the components that describe the energy which flows from systems $D$ to $C$, while for $N_{C}$ the direction is opposite. 


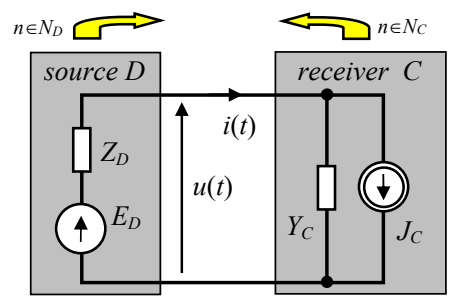

Where:

$N_{C} \in N, N_{D} \in N$

$N_{C} \neq N_{D}$

$N=N_{C} \cup N_{D}$

$Y_{C}$ - admittance of the $C$ system

$Z_{D}$ - internal impedance of the $D$ system

Fig. 2. A nonlinear receiver powered from a real source with many harmonics.

The active power associated with the energy flowing from the source to the receiver is the $P_{D(n)}$ positive power, and the negative power associated with the opposite direction is $P_{C(n)}$. In the real measuring system, the active power sign can be determined based on the knowledge of the phase shift angle $\varphi_{(n)}$ between the voltage and current harmonics:

$$
\left.\begin{array}{l}
\text { if }\left|\varphi_{(n)}\right| \leq 90^{0} \text { then } \quad P_{(n)} \geq 0 \\
\text { if }\left|\varphi_{(n)}\right|>90^{0} \text { then } P_{(n)}<0
\end{array}\right\} \begin{array}{ll}
P_{D(n)}=P_{(n)}, & n \in N_{D} \\
P_{C(n)}=-P_{(n)}, & n \in N_{C}
\end{array}
$$

When the active power $P_{(n)}$ for the $n$-th harmonic is positive, it should be assumed that it comes from the $D$ system. In a situation where the $E_{D}$ and $J_{C}$ sources have the same harmonics, one should check the direction of the flowing energy, i.e. which source is dominant.

Analogously to the division of active power, a division of current and voltage should be made. These components, related to the flow of energy from the source to the receiver, are as follows:

$$
i_{D}=\sum_{n \in N_{D}} i_{(n)}, \quad u_{D}=\sum_{n \in N_{D}} u_{(n)}, \quad P_{D}=\sum_{n \in N_{D}} P_{(n)},
$$

While for the opposite direction:

$$
i_{C}=\sum_{n \in N_{C}} i_{(n)}, \quad u_{C}=-\sum_{n \in N_{C}} u_{(n)}, \quad P_{C}=-\sum_{n \in N_{C}} P_{(n)} .
$$

This resulted in the distribution of variables into two subsets related to the direction of the flowing energy:

$$
i=i_{D}+i_{C}, \quad u=u_{D}-u_{C}, \quad P=P_{D}-P_{C} .
$$

$N_{C}$ and $N_{D}$ sets are disjoint, so the above components are mutually orthogonal:

$$
\|i\|^{2}=\left\|i_{D}\right\|^{2}+\left\|i_{C}\right\|^{2}, \quad\|u\|^{2}=\left\|u_{D}\right\|^{2}+\left\|u_{C}\right\|^{2}
$$

In [2], Czarnecki conducted a circuit analysis from Figure 2 in accordance with the superposition principle, i.e. he considered two phenomena existing independently. For each of these cases, the complex value of voltage can be determined:

$$
\underline{U}_{C(n)}=\frac{\underline{J}_{C(n)} \cdot \underline{Z}_{D(n)}}{1+\underline{Y}_{C(n)} \cdot \underline{Z}_{D(n)}}, \quad \text { for } n \in N_{C} .
$$

- the harmonics in the $i_{C}$ current caused by the $J_{C}$ source affect the $Z_{D}$ impedance only 
- the harmonics in the $i_{D}$ current caused by the $E_{D}$ source affect the $Y_{C}$ admittance only

$$
\underline{U}_{D(n)}=\frac{\underline{E}_{D(n)}}{1+\underline{Y}_{C(n)} \cdot \underline{Z}_{D(n)}}, \quad \text { for } n \in N_{D} .
$$

Equations (7) and (8) are correct for the different harmonics in the $E_{D}$ and $J_{C}$ sources. There may be a situation where the same harmonics will appear in the $E_{D}$ and $J_{C}$ sources. For example, this will be the case when two $C 1$ and $C 2$ nonlinear receivers (which produce the same harmonics) are connected to one $D$ system (Fig. 3.a).
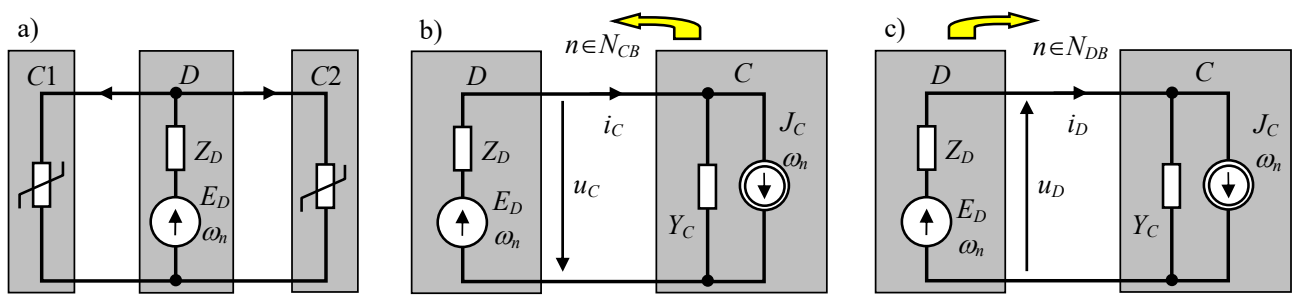

Fig. 3. Circuit that takes into account the same harmonics in the $E_{D}$ and $J_{C}$ sources.

A set of $N_{B}$ harmonics has been introduced containing such harmonics that occur in both sources at the same time. For $n \in N_{B}$ harmonics, the actual direction of the flowing energy will depend from the fact as to which source is dominant.
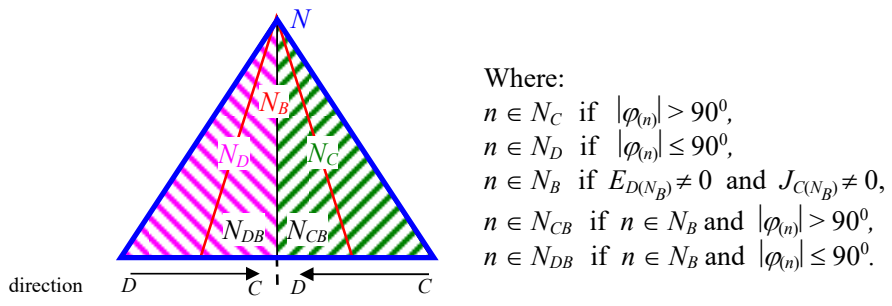

Fig. 4. Division of the set of $N$ harmonics into subsets of $N_{C}, N_{D}$ and $N_{B}$ due to the direction of flowing energy.

Harmonics occurring simultaneously in both $E_{D}$ and $J_{C}$ sources, and corresponding to the flowing energy from $D$ to $C$, are marked with the set $N_{D B}$, while the opposite (from $C$ to $D$ ) is marked $N_{C B}$, where $N_{B}=N_{D B} \cup N_{C B}$. For $n \in N_{B}$ harmonics, the complex voltage value is equal to:

$$
\underline{U}_{(n)}=\frac{\underline{E}_{D(n)}-\underline{J}_{C(n)} \cdot \underline{Z}_{D(n)}}{1+\underline{Y}_{C(n)} \cdot \underline{Z}_{D(n)}}, \quad \text { for } n \in N_{B}
$$

For the $N_{B}$ harmonics set, the direction of the flowing energy should be verified. This should be done based on Formula (2). Then, the $n$-th harmonics to the $N_{D B}$ or $N_{C B}$ set can be assigned.

According to the CPC theory, the $i_{D}$ current can be divided into the following components: active $i_{\mathrm{a}, D}$, scatter $i_{\mathrm{s}, D}$ and reactive $i_{\mathrm{r}, D}$ :

$$
i_{D}=i_{\mathrm{a}, D}+i_{\mathrm{s}, D}+i_{\mathrm{r}, D}
$$

A comparison of Formulas (5) and (10) results in the following: 


$$
i=i_{\mathrm{a}, D}+i_{\mathrm{s}, D}+i_{\mathrm{r}, D}+i_{C}
$$

All these components are mutually orthogonal, so it is correct to write:

$$
\|i\|^{2}=\left\|i_{\mathrm{a}, D}\right\|^{2}+\left\|i_{\mathrm{s}, D}\right\|^{2}+\left\|i_{\mathrm{r}, D}\right\|^{2}+\left\|i_{C}\right\|^{2}
$$

The power in this circuit requires knowledge of the energy properties of both the supplier and the recipient.

$S^{2}=\|u\|^{2}\|i\|^{2}=\left(\left\|u_{D}\right\|^{2}+\left\|u_{C}\right\|^{2}\right) \cdot\left(\left\|i_{D}\right\|^{2}+\left\|i_{C}\right\|^{2}\right)=\underbrace{\left\|u_{D}\right\|^{2}\left\|i_{D}\right\|^{2}}_{S_{D}^{2}}+\underbrace{\left\|u_{D}\right\|^{2}\left\|i_{C}\right\|^{2}+\left\|u_{C}\right\|^{2}\left\|i_{D}\right\|^{2}}_{S_{D C}^{2}}+\underbrace{\left\|u_{C}\right\|^{2}\left\|i_{C}\right\|^{2}}_{S_{C}^{2}}$

In this situation, the power equation takes the following form:

$$
S^{2}=P_{D}^{2}+D_{\mathrm{s}}^{2}+Q^{2}+S_{C}^{2}+S_{D C}^{2}
$$

Where the active power is equal to: $P=P_{D}-P_{C}$.

\section{EXAMPLE}

The nonlinear receiver has been connected to the real source according to Figure 2. System $D$ has the following parameters: $e_{D}=230 \sqrt{2} \sin \omega_{1} t+10 \sqrt{2} \sin 3 \omega_{1} t \mathrm{~V}, R_{D}=1 \Omega$, and $L_{D}=1 \mathrm{H}$. The non-linear receiver generates harmonics with a waveform: $j_{C}=10 \sqrt{2} \sin 3 \omega_{1} t+5 \sqrt{2} \sin 5 \omega_{1} t$ A. The $Z_{C}$ impedance of the receiver creates resistance $R_{C}=1 \Omega$ and inductance $L_{C}=1 \mathrm{H}$. The $e_{D}$ source with pulsation $\omega_{1}=1 \mathrm{rad} / \mathrm{s}$ causes the nonlinear receiver to generate the 3 rd and the 5 th harmonic. The 3 rd harmonic occurs in both sources $j_{C}$ and $e_{D}$. The impedance and admittance in both $C$ and $D$ systems for individual harmonics are as follows:

$$
\begin{array}{lll}
\underline{Z}_{D(1)}=R_{D}+j \omega_{(1)} L_{D}=(1+j 1) \Omega, & \underline{Z}_{D(3)}=(1+j 3) \Omega, & \underline{Z}_{D(5)}=(1+j 5) \Omega, \\
\underline{Y}_{C(1)}=\frac{1}{R_{C}+j \omega_{(1)} L_{C}}=\left(\frac{1}{2}-j \frac{1}{2}\right) \mathrm{S}, & \underline{Y}_{C(3)}=\left(\frac{1}{10}-j \frac{3}{10}\right) \mathrm{S}, & \underline{Y}_{C(5)}=\left(\frac{1}{26}-j \frac{5}{26}\right) \mathrm{S} .
\end{array}
$$

For the 1st harmonic, energy flows from $D$ to $C$, thus Formula (8) shows:

$\underline{U}_{(1)}=\underline{U}_{D(1)}=115 \mathrm{~V}, \underline{I}_{D(1)}=\underline{U}_{D(1)} \cdot \underline{Y}_{C(1)}=\left(\frac{115}{2}-j \frac{115}{2}\right) \mathrm{A}, P_{(1)}=\mathfrak{R} e\left\{\underline{U}_{D(1)} \cdot \underline{I}_{D(1)}^{*}\right\}=6612.5 \mathrm{~W}$.

For the 5th harmonic, the energy flow is opposite (from $C$ to $D$ ), therefore, it follows from Formula (7):

$\underline{U}_{(5)}=-\underline{U}_{C(5)}=-\left(\frac{5}{2}+j \frac{25}{2}\right) \mathrm{V}, \underline{I}_{C(5)}=-\frac{\underline{U}_{C(5)}}{\underline{Z}_{D(5)}}=-\frac{5}{2} \mathrm{~A}, \quad P_{(5)}=\mathfrak{R} e\left\{\underline{U}_{C(5)} \cdot \underline{I}_{C(5)}^{*}\right\}=-6.25 \mathrm{~W}$.

For the 3rd harmonic, Formula (9) is used:

$\underline{U}_{(3)}=-j 15 \mathrm{~V}, \underline{I}_{(3)}=\underline{U}_{(3)} \cdot \underline{Y}_{C(3)}=\left(-\frac{9}{2}-j \frac{3}{2}\right) \mathrm{A}, P_{(3)}=-22.5 \mathrm{~W}$.

The power for the $3 \mathrm{rd}$ harmonic is negative, so this harmonic belongs to the $N_{C B}$ set. These harmonics can be grouped as follows: $N=\{1,3,5\}, N_{D}=\{1\}, N_{C}=\{3,5\}, N_{C B}=\{3\}$.

Active powers are equal: $P_{D}=P_{(1)}, P_{C}=-P_{(3)}-P_{(5)}=28.75 \mathrm{~W}, P=P_{D}-P_{C}=6583.75 \mathrm{~W}$.

According to the CPC theory, the current components are as follows:

$\left\|i_{\mathrm{a}, D}\right\|=\frac{P_{D}}{\left\|u_{D}\right\|}=57.5 \mathrm{~A},\left\|i_{\mathrm{r}, D}\right\|=\sqrt{\sum_{n=1}\left(B_{(n)} U_{(n)}\right)^{2}}=57.5 \mathrm{~A},\left\|i_{C}\right\|=\sqrt{\sum_{n=3,5} I_{(n)}^{2}}=5.36 \mathrm{~A},\left\|i_{\mathrm{s}, D}\right\|=0 \mathrm{~A}$, 
The total current value is equal to: $\|i\|=\sqrt{\left\|i_{\mathrm{a}, D}\right\|^{2}+\left\|i_{\mathrm{s}, D}\right\|^{2}+\left\|i_{\mathrm{r}, D}\right\|^{2}+\left\|i_{C}\right\|^{2}}=81.49 \mathrm{~A}=\sqrt{\sum_{n \in N} I_{(n)}^{2}}$, and it is equal to the value determined from the sum of harmonics. Therefore, the decomposition for the current components was made correctly.

\section{Summary}

A change in the supply voltage $e_{D(n)}$ causes a change in the parameters of the nonlinear receiver: $J_{C}$ and $Y_{C}$. These parameters are functions that depend from value of the $u$ voltage. Thus, the analysis is true only at a specific work point. A circuit analysis for all types of nonlinear receivers is easier owing to formulas (7), (8), (9) presented in this article. By defining a new set of harmonics, it is possible to analyze circuits where harmonics are simultaneous in both systems. The proposed method is an extension of the CPC theory for single-phase circuits with nonlinear receivers. The conducted research has shown that the CPC power theory can be used to analyze circuits composed of several nonlinear receivers.

\section{References}

1. L.S. Czarnecki, Int. Journal on Circuit Theory and Appl. 11, 235-239 (1983)

2. L.S. Czarnecki, Moce $w$ obwodach elektrycznych $z$ niesinusoidalnymi przebiegami prądów i napięć, Warszawa (2005)

3. K. Zajkowski, EEMS Conf., E3S Web of Conferences, 19, 01037 (2017)

4. K. Zajkowski, EEMS Conf., E3S Web of Conferences, 19, 01033 (2017)

5. K. Zajkowski, Neural Computing and Appl., 24, 2, 431-439 (2014)

6. J. Smyczek, K. Zajkowski, Proc. 2nd International Industrial Simulation Conf., 278281 (2004)

7. S. Duer, K. Zajkowski, R. Duer, J. Paś, Neural Computing \& Appl., 23, 3-4, 913-925 (2013)

8. S. Duer, K. Zajkowski, I. Płocha, R. Duer, Neural Computing \& Appl., 22, 7, 15811590 (2013)

9. S. Duer, K. Zajkowski, Neural Computing \& Appl., 23, 7, 2185-2197 (2013)

10. K. Zajkowski, S. Scaticailov, Nonconventional Technologies Review, Romania, XX (2), 54-61 (2016)

11. K.Rokosz, T.Hryniewicz, Int. Journal of Materials Research, 104, 12 (2013)

12. L.S. Czarnecki, Harmonics and power phenomena, Wiley Encyclopedia of Electrical and Electronics Eng. (John Wiley \& Sons 2000) 